\title{
Connection between the Spectrum Condition and the Lorentz Invariance of the Yukawa 2 Quantum Field Theory
}

\section{Edward P. Osipov}

Department of Theoretical Physics, Institute for Mathematics, 630090, Novosibirsk, 90, USSR

\begin{abstract}
We prove, under assumptions, the Lorentz invariance of some quantum field theories. In the separate paper we show that our assumptions are fulfilled in the (renormalized) Yukawa ${ }_{2}$ quantum field theory with the periodic boundary conditions.
\end{abstract}

In the present paper we prove, under several assumptions, the Lorentz invariance of some quantum field theories. In a separate paper we show that our assumptions are fulfilled for the CPT invariant states in the (renormalized) Yukawa ${ }_{2}$ quantum field theory with the periodic boundary conditions (see also [1]). Keeping this application in mind, we bound ourselves to the case of the Yukawa ${ }_{2}$ quantum field theory.

For the sake of convenience, we introduce a space of four-component complexvalued test functions and we set for each four-component function

$$
\Psi_{0}(h):=\sum_{\alpha=1}^{2} \int d x \psi_{0}^{\alpha}(x) h_{\alpha}(x)+\sum_{\alpha=3}^{4} \int d x \bar{\psi}_{0}^{\alpha-2}(x) h_{\alpha}(x)
$$

where $\psi_{0}^{\alpha}(x)$ is the free fermion field in the one-dimensional space and $\overline{\psi_{0}}(x)=\psi_{0}(x)^{*} \gamma_{0}$. If $h(x)$ is a four-component function, we set

$$
\operatorname{supp} h:=\bigcup_{\alpha} \operatorname{supp} h_{\alpha}(x) \text {. }
$$

Let $\mathfrak{U}_{f}$ be the field algebra for the $Y_{2}$, as it is defined in [2], i.e., $\mathfrak{A}_{f}$ is the $C^{*}$ algebra defined as the norm closure of $\bigcup_{B} \mathfrak{A}_{f}(B)$, where $\mathfrak{A}_{f}(B)$ is the von Neumann algebra generated by bounded functions of the time zero free scalar field $\varphi_{0}(f)$ and its time derivative $\pi_{0}(f), f \in C_{0}^{\infty}(\mathbb{R}), \operatorname{supp} f \subset B$, and by the operators of the free fermion field $\Psi_{0}(h), h \in C_{0}^{\infty}(\mathbb{R}) \otimes \mathbb{C}^{4}$, supp $h \subset B$.

Let $\mathscr{P}_{2}$ be the Poincare group (= the inhomogeneous Lorentz group) in two space-time dimensions, i.e. the three-parameter group of transformations

$$
\begin{aligned}
\left\{a_{0}, a_{1}, \lambda\right\}(t, x)= & \left(a_{0}+\frac{1}{2}\left(\lambda^{2}+\lambda^{-2}\right) t+\frac{1}{2}\left(\lambda^{2}-\lambda^{-2}\right) x,\right. \\
& \left.a_{1}+\frac{1}{2}\left(\lambda^{2}-\lambda^{-2}\right) t+\frac{1}{2}\left(\lambda^{2}+\lambda^{-2}\right) x\right) .
\end{aligned}
$$


Assumption 1. (Existence of the dynamics.) We suppose that there exists a representation $\sigma\left(\left\{a_{0}, a_{1}, \lambda\right\}\right)$ of the group $\mathscr{P}_{2}$ by automorphisms of the algebra $\mathfrak{A}_{f}$ such that the spatial translations coincide with the free ones $\exp (i P x)(\cdot) \exp (-i P x)$. Let us denote $\sigma_{t}=\sigma(\{t, 0,0\})$. We suppose that for each positive $b$, there exists a self-adjoint operator $H(b)$ in the Fock space, where the algebra $\mathfrak{U}_{f}$ is defined, such that for $A \in \mathfrak{A}_{f}(B), B \subset[-b, b]$ and $|t|<b$

$$
\sigma_{t}(A)=\exp (i t H(b)) A \exp (-i t H(b)) \text {. }
$$

Assumption 2. (Locality.) We suppose that the automorphisms $\sigma_{t}$ are local, i.e., that one of the following conditions is satisfied:

1) $\sigma_{t}(\mathfrak{A}(B)) \subset \mathfrak{A}\left(B_{t}\right)$

where $B_{t}$ is the set of points within distance $|t|$ of $B$.

2) Let $\mathscr{B}(B)\left(\subset \mathfrak{A}_{f}(B)\right)$ be the subalgebra of bounded boson observables localized in the region $B$. If $(t, B)$ and $\left(t^{\prime}, B^{\prime}\right)$ are spacelike separated and $\operatorname{supp} h \subset B, \operatorname{supp} h^{\prime} \subset B^{\prime}$, then

$$
\begin{aligned}
& {\left[\sigma_{t}(\mathscr{B}(B)), \sigma_{t^{\prime}}\left(\mathscr{B}\left(B^{\prime}\right)\right)\right]=0,} \\
& {\left[\sigma_{t}\left(\Psi_{0}(h)\right), \sigma_{t^{\prime}}\left(\mathscr{B}\left(B^{\prime}\right)\right)\right]=0,} \\
& \left\{\sigma_{t}\left(\Psi_{0}(h)\right), \sigma_{t^{\prime}}\left(\Psi_{0}\left(h^{\prime}\right)\right)\right\}=0 .
\end{aligned}
$$

Remark. We note that 2.1) implies 2.2).

Assumption 3. (Covariance.) For $f \in C_{0}^{\infty}\left(\mathbb{R}^{2}\right) \otimes \mathbb{C}^{4}$ we set

$$
\Psi(f):=\int d t \sigma_{t}\left(\Psi_{0}(f(t, \cdot))\right) \in \mathfrak{A}_{f} .
$$

We suppose that for each $f \in C_{0}^{\infty}\left(\mathbb{R}^{2}\right) \otimes \mathbb{C}^{4}$

$$
\sigma\left(\left\{a_{0}, a_{1}, \lambda\right\}\right) \Psi(f)=\Psi\left(S(\lambda) f\left(\left\{a_{0}, a_{1}, \lambda\right\}(t, x)\right)\right)
$$

where $S(\lambda)$ is the $4 \times 4$ matrix

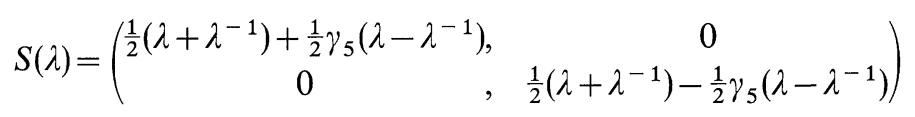

and

$$
\gamma_{5}=\left(\begin{array}{cc}
1 & 0 \\
0 & -1
\end{array}\right)
$$

Assumption 4. (Existence of the physical Hilbert space.) We suppose that there exists a translation invariant (under $\sigma\left(\left\{a_{0}, a_{1}, 0\right\}\right)$ ) state $\omega$ on the algebra $\mathfrak{U}_{f}$, which is locally Fock. (For a definition of the locally Fock property see [3].)

Let $\left(\mathscr{H}_{\omega}, \pi_{\omega}, U_{\omega}(a), \Omega_{\omega}\right)$ be the Gelfand-Naimark-Segal representation corresponding to the state $\omega$. As in [3], Assumptions 1 and 4 imply that $U_{\omega}(a)$ are strongly continuous in the Hilbert space $\mathscr{H}_{\omega}$. Let $H_{\omega}$ and $P_{\omega}$ be the energy and momentum operators in the GNS representation.

Assumption 5. (Spectral condition.) We suppose that

$$
H_{\omega} \geqq 0, \quad H_{\omega}^{2} \geqq P_{\omega}^{2} .
$$


Assumption 4 implies that the group of the unitary operators $\pi_{\omega}\left(\exp \left(i s \varphi_{0}(f)\right)\right)$ for real $f \in C_{0}^{\infty}(\mathbb{R})$ is weakly continuous in $\mathscr{H}_{\omega}$ in $s$. Let $\varphi_{0}(f)_{\omega}$ be the corresponding self-adjoint generator.

Assumption 6. $\left(\varphi, \pi\right.$-bounds.) Let $f \in C_{0}^{\infty}(\mathbb{R})$, we suppose that

a) $\left|\varphi_{0}(f)_{\omega}\right| \leqq\|f\|_{\mathscr{S}}\left(H_{\omega}+1\right)$;

b) $\pm \varphi_{0}(f)_{\omega} \leqq c\|f\|_{1}\left(H_{\omega}+1\right)$;

c) $\pm i\left[\varphi_{0}(f)_{\omega}, H_{\omega}\right] \leqq c\|f\|_{2}\left(H_{\omega}+1\right)$,

as bilinear forms on $\mathscr{D}^{\infty}\left(H_{\omega}\right) \times \mathscr{D}^{\infty}\left(H_{\omega}\right)$. Here $\|\cdot\|_{\mathscr{S}}$ is a norm on the Schwartz space $\mathscr{S}(\mathbb{R})$ and $\|\cdot\|_{p}$ is the $L_{p}(\mathbb{R})$ norm.

Assumption 7. (CPT invariance.) We suppose that there exists an antiautomorphism $\vartheta$ of the algebra $\mathfrak{A}_{f}$, such that

$$
\vartheta \sigma\left(\left\{a_{0}, a_{1}, \lambda\right\}\right)=\sigma\left(\left\{-a_{0},-a_{1}, \lambda\right\}\right) \vartheta .
$$

We suppose that the state $\omega$ (Assumption 4) is $\vartheta$ invariant, that is, $\omega(\vartheta(A))=\omega(A)^{*}$ $=\omega\left(A^{*}\right)$ for all $A \in \mathfrak{A}_{f}$.

Theorem. Under Assumptions 1-7 the state $\omega$ is Poincare invariant. The corresponding Wightman functions exist as distributions and are Poincare covariant.

Proof of the Theorem. Assumption 6a and Proposition 1 by Nelson [4] imply that the vacuum expectation values of any number of smeared field operators [with test functions from $C_{0}^{\infty}\left(\mathbb{R}^{2}\right)$ or $\left.C_{0}^{\infty}\left(\mathbb{R}^{2}\right) \otimes \mathbb{C}^{4}\right]$ are well-defined. If $f \in C_{0}^{\infty}\left(\mathbb{R}^{2}\right)$, then on the domain $\mathscr{D}^{\infty}\left(H_{\omega}\right)$

$$
\begin{aligned}
\varphi(f): & =\int d t \exp \left(i t H_{\omega}\right) \varphi_{0}(f(t, \cdot))_{\omega} \exp \left(-i t H_{\omega}\right) \\
& \leqq \int d t\|f(t, \cdot)\|_{\mathscr{S}(\mathbb{R})}\left(H_{\omega}+1\right) \leqq\|f\|_{\mathscr{S}\left(R^{2}\right)}\left(H_{\omega}+1\right) .
\end{aligned}
$$

The arguments of Glimm and Jaffe [5] (or the repeated application of Nelson's lemma [4]) show that the vacuum expectation values are continuous on the Schwartz space. Since they are linear functionals (this easily follows from the locally Fock property of the state $\omega$ ), so the Schwartz nuclear theorem proves that the vacuum expectation values are tempered distributions.

As in Proposition 1.1 by Glimm and Jaffe [5] one can show that the Hilbert spaces $\mathscr{H}_{W}$ and $\mathscr{H}_{\omega}$, constructed by the Wightman reconstruction theorem and by GNS construction coincide. This implies that to prove the Poincare invariance of the state it is sufficient to prove the Poincare covariance of the Wightman functions.

Now we proceed to the proof of the Poincare covariance of the Wightman functions.

Assumptions 4 and 5 imply that the Wightman functions are translation invariant and satisfy the spectrum condition. Assumption 2 implies that the Wightman functions satisfy the local (anti)commutativity. Since the Wightman functions are translation invariant, they define the sequence of the distributions, depending on the differences of coordinates. Each such distribution satisfies the conditions of the last theorem, Chapter IV, $\$ 7$ of Jost's book [6] and so is the boundary value of a function holomorphic in the extended tube, having a onevalued continuation into the union of the permuted extended tubes [6]. 
Let $W\left(z_{1}, \ldots, z_{n}\right)(z:=(t, x))$ be a Wightman function of $n$ fields (scalar or fermion) and we do not write down fermion indices. Let $W\left(\lambda, z_{1}, \ldots, z_{n}\right)$ be the distribution defined by

$$
\left\langle W\left(\lambda, z_{1}, \ldots, z_{n}\right), f_{1} \ldots f_{n}\right\rangle=\left\langle W\left(z_{1}, \ldots, z_{n}\right), f_{1}(\lambda) \ldots f_{n}(\lambda)\right\rangle
$$

$\langle>$ denotes the integrating over the space and time variables and the summation over the fermion variables and $f(\lambda)=f(\{0,0, \lambda\} z)$. The distributions $W\left(\lambda, z_{1}, \ldots, z_{n}\right)$ have a one-valued continuation into the union of permuted extended tubes and so satisfy the hypotheses of the theorem on finite covariance of Bros et al. [7,8] (which is valid in the two-dimensional case also), and so $W\left(\lambda, z_{1}, \ldots, z_{n}\right)$ has the unique expansion

$$
W\left(\lambda, z_{1}, \ldots, z_{n}\right)=\sum_{-N^{-} \leqq k \leqq N^{+}, k \text { even }} \lambda^{k} W^{k}\left(z_{1}, \ldots, z_{n}\right)
$$

where $W^{k}\left(z_{1}, \ldots, z_{n}\right)$ is a tempered distribution of tensor rank $k$, and is the boundary value of a function holomorphic in the extended tube, having a one-valued continuation into the union of the permuted extended tubes [6]. We note that the sum in (1) is taken only over even $k$.

Now let $W^{S}\left(\lambda, z_{1}, \ldots, z_{n}\right)$ be the distributions defined by

$$
\left\langle W^{S}\left(\lambda, z_{1}, \ldots, z_{n}\right), f_{1} \ldots f_{n}\right\rangle=\left\langle W\left(z_{1}, \ldots, z_{n}\right), f_{1}^{S}(\lambda) \ldots f_{n}^{S}(\lambda)\right\rangle
$$

where

$$
f^{s}(\lambda)=\left\{\begin{array}{l}
f(\{0,0, \lambda\} z) \text { for the scalar field } \\
S(\lambda) f(\{0,0, \lambda\} z) \text { for the fermion field } .
\end{array}\right.
$$

From the expansion (1) and the explicit form of $S(\lambda)$ it follows that $W^{S}(\lambda, \cdot)$ also has the unique expansion of the type (1)

$$
W^{S}(\lambda, \cdot)=\sum_{-N^{-} \leqq k \leqq N^{+}} \lambda^{k} W^{k}(\cdot) .
$$

Here the sum is taken over integer $k$ (even and odd).

To prove the Poincare covariance it is sufficient to prove that in the expansion (2) $N^{ \pm}=0$ for each distribution $W^{S}(\lambda, \cdot)$.

Let $N^{ \pm}(m, n, l)$ be the maximal degree of $\lambda^{ \pm 1}$, which enters the expansion (2), for a Wightman function with $m$ scalar fields, with $n$ fields which are the time derivative of the scalar field and with $l$ fermion fields. Because of local (anti) commutativity, $N^{ \pm}(m, n, l)$ are independent of the order among fields.

The following lemma reduces the proof of the Poincare covariance of the whole theory to the proof of the Poincare invariance of its scalar part.

Lemma. $N^{ \pm}(m, 0, l) \leqq N^{ \pm}(2 m, 0,0)$.

Proof of the Lemma. Let $W_{m, 0, l}^{N^{ \pm}}(\cdot)$ be the part of $W_{m, 0, l}^{S}(\lambda, \cdot)$ of highest rank $N^{ \pm}(m, 0, l)$. Let

$$
\begin{aligned}
& f_{j, \pm} \in C_{0}^{\infty}\left(\mathbb{R}^{2}\right), \quad j=1,2, \ldots, m, \\
& f_{j, \pm} \in C_{0}^{\infty}\left(\mathbb{R}^{2}\right) \otimes \mathbb{C}^{4}, \quad j=m+1, \ldots, m+l
\end{aligned}
$$

be chosen with mutually space-like supports and

$$
\left\langle W_{m, 0, l}^{N^{ \pm}}, \otimes f_{j, \pm}\right\rangle>0 \text {. }
$$


Since $W_{m, 0, l}^{N^{ \pm}}$are holomorphic in the union of the permuted extended tubes, including Jost points, the analyticity and Bogoliubov's edge of the wedge theorem [9] imply that such $f_{j, \pm}$ exist (otherwise $W_{m, 0, l}^{N^{ \pm}}=0$ ).

Then Schwarz's inequality gives

$$
\begin{aligned}
& \left\langle W_{m, 0, l}^{S}(\lambda, \cdot), \otimes f_{j, \pm}\right\rangle \\
& \quad=\left(\Omega_{\omega}, \varphi\left(f_{1, \pm}^{S}(\lambda)\right) \ldots \varphi\left(f_{m, \pm}^{S}(\lambda)\right) \Psi\left(f_{m+1, \pm}^{S}(\lambda)\right) \ldots \Psi\left(f_{m+l, \pm}^{S}(\lambda)\right) \Omega_{\omega}\right) \\
& \quad \leqq\left\|\varphi\left(f_{m, \pm}^{S}(\lambda)\right)^{*} \ldots \varphi\left(f_{1, \pm}^{S}(\lambda)\right)^{*} \Omega_{\omega}\right\| \cdot\left\|\Psi\left(f_{m+1, \pm}^{S}(\lambda)\right) \ldots \Psi\left(f_{m+l, \pm}^{S}(\lambda)\right) \Omega_{\omega}\right\| \\
& \leqq c|\lambda|^{ \pm N^{ \pm}(2 m, 0,0)} \omega\left(\sigma(\{0,0, \lambda\})\left(\prod_{j=l}^{1} \Psi\left(f_{m+j, \pm}\right)^{*} \prod_{j=1}^{l} \Psi\left(f_{m+j, \pm}\right)\right)\right)^{1 / 2} \\
& \leqq c|\lambda|^{ \pm N^{ \pm}(2 m, 0,0)} \prod_{j=1}^{l}\left\|\Psi\left(f_{m+j, \pm}\right)\right\|^{2} \\
& \leqq\left. c\right|_{1}|\lambda|^{ \pm N^{ \pm}(2 m, 0,0)} .
\end{aligned}
$$

We use the following simple estimate

$$
\begin{aligned}
\|\Psi(f)\| & \leqq \int d t\left\|\sigma_{t}(\Psi(f(t, \cdot)))\right\| \leqq \int d t\|\Psi(f(t, \cdot))\| \\
& \leqq \int d t\|f(t, \cdot)\|_{2}=\int d t\left(\sum_{\alpha}\left(\int d x\left|f_{\alpha}(t, x)\right|^{2}\right)^{1 / 2}\right)
\end{aligned}
$$

Since the left-hand side has leading term $\lambda^{ \pm N^{ \pm}(m, 0, l)}$ with non-zero coefficient, so the lemma is proved.

So to prove the Poincare covariance it is sufficient to prove the Poincare invariance for the Wightman functions of the scalar field. But for the Wightman functions of the scalar field the Poincare invariance may be proved in the same way as it has been done by Streater for the $P(\varphi)_{2}$ model [10].

In fact, the bounds $6 \mathrm{~b}$ ), c) coincide with the bounds (2), (1) of Streater [10] and Assumption 6 implies the bounds of the type (11), (21) of Streater [10]. Further, Streater has used PT invariance of the $P(\varphi)_{2}$ theory to prove that his $N^{ \pm}(0, n)$ is even (for even $n$ ). In fact, this assertion follows from the CPT invariance. Really, the hermiticity of the scalar field implies that $W_{0, n, 0}(\lambda, z)=W_{0, n, 0}(\lambda, z)^{*}$ in Jost points. In addition, $W_{0, n, 0}(\lambda, z)$ is a function of $\lambda^{2}$ [the expansion (1) contains only the even degrees of $\lambda]$. The CPT invariance (Assumption 7) gives $W_{0, n, 0}(\lambda, z)$ $=(-1)^{n} W_{0, n, 0}(\lambda,-z)^{*}$. This equality and the above arguments show that for even $n$ $W_{0, n, 0}(\lambda, z)$ is an even function of $\lambda^{2}$, i.e., that our $N^{ \pm}(0, n, 0)$ is a multiple of four.

Using this remark and Streater's arguments we obtain $N^{ \pm}(m, 0,0)=0$ and so $0 \leqq N^{ \pm}(m, 0, l) \leqq N^{ \pm}(2 m, 0,0)=0$. The theorem is proved.

Now we prove the natural consequence.

Corollary. The Wightman functions involving an odd number of the fermion fields are zero.

Proof of the Corollary. Let $W(z)$ be the Wightman function with an odd number of the fermion fields. The expansion (1) implies that $W(\lambda, z)$ is an even function of $\lambda$, but the Poincare covariance implies that $W(\lambda, z)=\left(\prod S(\lambda)^{-1}\right) W(z)$, i.e., that $W(\lambda, z)$ is an odd function of $\lambda$. Thus, $W(\lambda, z)=0$ and $W(z)=0$. The corollary is proved. 
Remark. We note that our proof uses the existence of the automorphism $\sigma(\{0,0, \lambda\})$ for the fermion field only. The Lorentz invariance of the scalar part is contained, in fact, in the estimates of Assumptions 6b), c). Also, the CPT invariance of Wightman functions is needed only for even number of scalar fields.

Acknowledgements. The author is indebted to E. Heifets for communication and discussion of his results prior to publication. It is a pleasure to thank the Budnev brothers for the valuable advice.

\section{References}

1. Heifets,E.P., Osipov,E.P.: The energy-momentum spectrum in the Yukawa ${ }_{2}$ quantum field theory. Commun. math. Phys. 57, 31-50 (1977)

2. Glimm, J., Jaffe, A.: The Yukawa ${ }_{2}$ quantum field theory without cutoffs. J. Funct. Anal. 7, 323-357 (1971)

3. Glimm, J., Jaffe, A. : The $\lambda\left(\varphi^{4}\right)_{2}$ quantum field theory without cutoffs. III. The physical vacuum. Acta Math. 125, 203-267 (1970)

4. Nelson,E.: Time-ordered products of sharp-time quadratic forms. J. Funct. Anal. 11, 211-219 (1972)

5. Glimm,J., Jaffe, A.: The $\lambda \varphi_{2}^{4}$ quantum field theory without cutoffs. IV. Perturbations of the Hamiltonian. J. Math. Phys. 13, 1568-1584 (1972)

6. Jost,R.: The general theory of quantized fields. Providence R.I.: Amer. Math. Soc. Publ. 1965

7. Bros,J., Epstein,H., Glaser,V.: On the connection between analyticity and Lorentz covariance of Wightman functions. Commun. math. Phys. 6, 77-100 (1967)

8. Bogoliubov, N.N., Vladimirov, V.S.: Representations of $n$-point functions (In Russian). In: Trudi Matematicheskogo Instituta imeni Steklova, Vol. 112, pp. 5-21. Moscow: Nauka 1971

9. Vladimirov,V.S.: Methods of the theory of functions of several complex variables. Cambridge and London: MIT Press 1966

10. Streater, R.F.: Connection between the spectrum condition and the Lorentz invariance of $P(\varphi)_{2}$. Commun. math. Phys. 26, 109-120 (1972)

Communicated by A. Jaffe

Received April 8, 1977 